\title{
Mycobacterial infection in HIV seropositive and seronegative populations, 1987-93
}

\author{
Ian K Taylor, David J Evans, Richard J Coker, David M Mitchell, Rory J Shaw
}

\begin{abstract}
Background - Although the causes of the worldwide resurgence of tuberculosis are multifactorial, the HIV epidemic is believed to have had a central role. Control is further threatened by the emergence of multidrug-resistant tuberculosis.

Methods - A retrospective evaluation was undertaken of trends in pulmonary and extrapulmonary culture positive mycobacterial pathology, and the prevalence of drug-resistant tuberculosis in both HIV seropositive and, presumptively, HIV seronegative patients receiving their clinical care at St Mary's Hospital, London. Five hundred and thirty eight patients (188 of whom were known to be HIV seropositive) with positive mycobacterial isolates between January 1987 and March 1993 were identified from laboratory records. These were cross referenced with drug surveillance records.
\end{abstract}

Results - Overall, between 1987 and 1992 there was a progressive 3.5 fold increase in positive mycobacterial isolates and $a$ $2 \cdot 5$ fold increase in patients with proven mycobacterial infection. This increase was greater within the HIV seropositive population. A total of 663 positive mycobacterial isolates was evaluated; the major pathogen identified was Mycobacterium tuberculosis (379 isolates, 57\%). Three hundred and fourteen patients were diagnosed as having $M$ tuberculosis, 49 of whom were HIV seropositive. M tuberculosis was predominantly isolated from the lung. Of 358 positive cultures for $M$ tuberculosis (68 HIV seropositive, 290 presumptively HIV seronegative), only 27 isolates $(7 \cdot 6 \%)$, almost exclusively derived from presumed HIV seronegative patients, were resistant to either isoniazid, rifampicin, or both drugs together. No increases in drug-resistant isolates were observed over this period.

Conclusions - There has been a considerable increase in the incidence of tuberculosis in both HIV seronegative and seropositive populations during the study period. The emergence of drug-resistant tuberculosis was not observed.

(Thorax 1995;50:1147-1150)

Keywords: tuberculosis, HIV infection, drug resistance.

The worldwide resurgence of tuberculosis has been attributed to many factors. Whilst the HIV epidemic is believed to have had a central role, ${ }^{1-3}$ other issues such as poverty, homelessness, immigration, and limited access to medical care have also contributed to the increase.$^{45}$ Control is further threatened by the emergence of multidrug-resistant tuberculosis. ${ }^{6}$

St Mary's Hospital in north west London serves an inner city population at the lower end of the socioeconomic spectrum which has a high proportion of ethnic groups at risk of developing mycobacterial disease. Since 1983 St Mary's Hospital has been one of the largest HIV referral centres in the UK and in the period to March 19931956 patients with HIV infection, including 914 with AIDS, have received their regular clinical management. The principal aim of this study was a retrospective evaluation of trends in mycobacterial disease in both known HIV seropositive and presumptively HIV seronegative patients over the 75 month period from January 1987 to March 1993. The primary point of data capture was from the laboratory records of positive mycobacterial isolates of patients undergoing clinical investigation for suspected pulmonary or systemic disease; as such, these data represent routine clinical practice. As a secondary aim, potential emergence of drug-resistant tuberculosis has also been assessed in both patient subpopulations by cross referencing with drug surveillance records.

\section{Methods}

All patients investigated at St Mary's Hospital between January 1987 and March 1993 and who had proven culture positive mycobacterial pathology were evaluated. The data source was mycobacterial laboratory records in which positive isolates were identified. Histological records were not assessed as the database was only accurate for culture of specimens and the frequency of documentation of histological reports not adequate to allow reliable conclusions. Information on the age, sex, HIV status, and site of isolation of the mycobacterial organism was obtained from the laboratory records. The site of isolation of the mycobacterial organism was arbitrarily divided into those of pulmonary origin (sputum, bronchoalveolar lavage fluid, pleural biopsy specimen, pleural fluid, or bronchial biopsy specimen) and those of extrapulmonary origin. For the purposes of this study repeat positive cultures of the same mycobacterial pathogen from the same anatomical site within nine months of the initial isolate were not recorded. All cases of smear positive pulmonary $M$ tuberculosis obtained from sputum or bronchoalveolar lavage fluid were recorded. Following initial identification of positive mycobacterial cultures, 


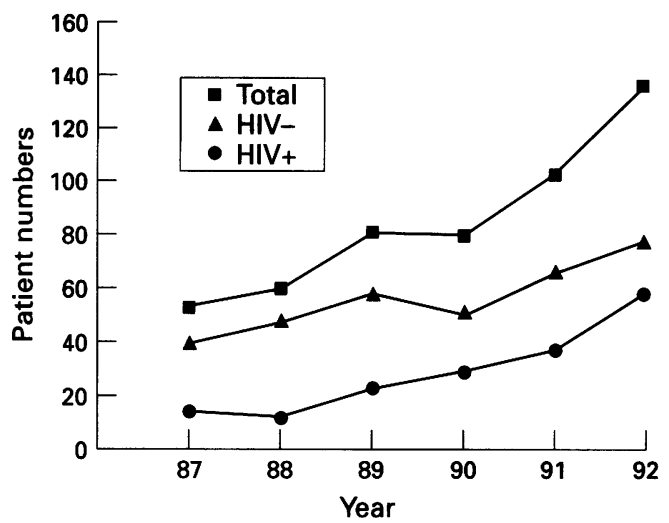

Number of patients with mycobacterial infection 1987-92.

specimens were sent to the Mycobacterium Reference Unit in Cardiff. Mycobacteria were identified by routine cultural and biochemical tests $^{7}$ and in vitro drug susceptibility was evaluated. The putative clinical significance of the mycobacteria isolated was not a primary aim of this study. Thus, the laboratory findings were not correlated with clinical symptomatology, radiographic evaluation or, in the HIV seropositive population, surrogate markers of disease progression such as CD4 counts. Patients with positive mycobacterial isolates were not prospectively tested for HIV disease.

\section{Results}

During the 75 month period of the study (January 1987 to March 1993) 538 patients with culture positive mycobacterial disease were diagnosed. Their age ranged from five to 89 years and 402 were men. One hundred and eighty eight were known to be HIV seropositive. Amongst the HIV seropositive population (age range 13-50 years) only three were women; most $(97 \%)$ were homosexual or bisexual men.

Between 1987 and 1992 there were progressive annual increases in both the number of patients with mycobacterial infection (figure) and the number of positive isolates of 2.5 and 3.5 fold, respectively. Within the HIV seronegative subpopulation there was an approximate twofold increase in both patient numbers and positive isolates. By contrast, in the HIV seropositive population there was a fivefold increase in positive mycobacterial cultures and a fourfold increase in patient numbers. These results were in parallel with an increase in the number of patients and positive isolates for tuberculous infection.

The total number of cultures positive for mycobacteria in this study was 663,379 derived from 350 patients presumptively negative for HIV infection and 284 from the known 188 patients with HIV disease (table 1). The predominant pathogen amongst those patients who were HIV seronegative was $M$ tuberculosis, largely of pulmonary origin. The total number of patients with culture positive $M$ tuberculosis was 314 (that is, $M$ tuberculosis alone or in association with another mycobacterial organism), 49 of whom were known to have HIV infection. Of the 265 patients presumptively HIV seronegative with tuberculosis, the patho-
Table 1 Mycobacterial pathogen isolated from both HIV seropositive and seronegative populations, Fanuary 1987 to March 1993

\begin{tabular}{|c|c|c|c|}
\hline & $H I V+$ & $H I V-$ & $\begin{array}{l}\text { All } \\
\text { patients }\end{array}$ \\
\hline $\begin{array}{l}\text { Patients with } M \text { tuberculosis alone } \\
\text { Patients with } M \text { avium- }\end{array}$ & 43 & 265 & 308 \\
\hline & 91 & 44 & 135 \\
\hline $\begin{array}{l}\text { non-tuberculous mycobacteria } \\
\text { (not MAI) }\end{array}$ & 43 & 39 & 82 \\
\hline $\begin{array}{l}\text { Patients with } M \text { tuberculosis and } \\
\text { one other atypical mycobacteria } \\
\text { Patients with two different atypical }\end{array}$ & 6 & 0 & 6 \\
\hline $\begin{array}{l}\text { non-tuberculous mycobacteria } \\
\text { Patients with unclassified }\end{array}$ & 5 & 1 & 6 \\
\hline $\begin{array}{l}\text { mycobacteria } \\
\text { Total }\end{array}$ & $\begin{array}{r}1 \\
188\end{array}$ & $\begin{array}{r}0 \\
350\end{array}$ & $\begin{array}{r}1 \\
538\end{array}$ \\
\hline
\end{tabular}

Table 2 Culture positive mycobacterial infection delineated by species and site, fanuary 1987 to March 1993

\begin{tabular}{lc}
\hline & Total \\
\hline Patients with positive mycobacterial pathology & 538 \\
Patients with positive mycobacterial pathology & \\
known HIV seropositive & 188 \\
Patients with positive mycobacterial pathology & \\
presumptively HIV seronegative & 350 \\
Number of positive mycobacterial cultures in HIV & \\
seropositive population & 284 \\
Number of positive mycobacterial cultures in HIV & \\
seronegative population & 379 \\
Pulmonary cultures & \\
Total & 361 \\
$M$ tuberculosis (HIV-) & 192 \\
$M$ tuberculosis (HIV +) & 34 \\
Pulmonary non-tuberculous spp (HIV-) & 45 \\
Pulmonary non-tuberculous spp (HIV +) & 90 \\
& \\
Extrapulmonary cultures & 302 \\
Total & 98 \\
Extrapulmonary $M$ tuberculosis (HIV-) & 34 \\
Extrapulmonary $M$ tuberculosis (HIV+) & 44 \\
Extrapulmonary non-tuberculous spp (HIV-) & 126 \\
Extrapulmonary non-tuberculous spp (HIV +) & \\
\hline
\end{tabular}

gen was isolated from the lung in 172, from extrapulmonary sources in 85 , and from both pulmonary and extrapulmonary sites in eight. In the 49 patients with HIV infection and tuberculosis the pathogen was isolated from the lung in 21 patients, from extrapulmonary sites in 17, and from both pulmonary and extrapulmonary sites in 11 patients. The analysis of positive cultures is shown in table 2.

With respect to atypical isolates, $M$ avium was the predominant organism (205 of 663 cultures, $77 \%$ of which were from HIV seropositive patients); however, $M$ fortuitum, $M$ xenopi, and $M$ kansasii were occasionally isolated (78 of 663 cultures, $61 \%$ of which were from HIV seropositive patients) and were the only other organisms that were cultured on more than a sporadic basis.

\section{DRUG SENSITIVITIES}

Over the 75 month period of the study 358 cultures positive for $M$ tuberculosis were identified of which only $27(7 \cdot 6 \%)$ exhibited resistance to either isoniazid, rifampicin, or both drugs together. Resistance to isoniazid alone amongst all tuberculous isolates was $4.5 \%$, to rifampicin alone $0.3 \%$, and to both drugs in the same culture $2 \cdot 8 \%$. The annual incidence of resistance to these drugs over the period of the study showed no appreciable progressive increase. Of the 24 patients in whom resistance to these drugs was demonstrated, representing 
$4.5 \%$ of the total patient population, 15 were recent immigrants ( $<5$ years), two were intravenous drug abusers, six had a history of previous antituberculous treatment, and only two patients were known to be HIV seropositive.

\section{PREVALENCE OF MYCOBACTERIAL DISEASE IN} HIV SEROPOSITIVE PATIENTS

The total number of new patients registered with HIV infection at St Mary's Hospital since the beginning of the HIV epidemic in 1983 is 1956. If all patients with known HIV disease since 1983 are included, the proportion of patients with mycobacterial disease based on the data in this study could be estimated at $9 \cdot 6 \%$ (188 of 1956). This is likely to represent an underestimation since the number of HIV seropositive patients with positive mycobacterial isolates between January 1983 and December 1986 has not been evaluated in this study. If only notification records had been used the incidence of tuberculosis over this period would be four of $1441(0 \cdot 28 \%$; data not shown).

\section{Discussion}

There are three principal findings relating to this study. Firstly, between 1987 and 1992 there was a progressive increase in positive mycobacterial isolates and patients with mycobacterial disease in both HIV seropositive and presumptively seronegative subpopulations. This increase was greater in the HIV seropositive population. Even so, our data may well represent an underestimation of diagnostic rates since patients in both subpopulations diagnosed on the basis of histopathology alone were not included. Our data may relate not only to an increase in the incidence of mycobacterial disease with time, but also to an increase in the number of patients within at risk groups in the local population. Secondly, as anticipated, the major mycobacterial pathogen in presumptive HIV seronegative patients was $M$ tuberculosis which was principally pulmonary in origin. Thirdly, in contrast to urban areas of the USA where drug resistance is reported to be as high as $33 \%$, 6 our data do not show an emergence of drug-resistant tuberculosis in inner London with an incidence of only $4.5 \%$. In the patients in our study in whom drug resistance was demonstrated, immigration, drug abuse, and previous antituberculous therapy may have been contributory. Only two of these patients were known to be HIV seropositive.

In the USA it has been estimated that up to $12 \%$ of patients with tuberculosis are seropositive for HIV infection. ${ }^{8}$ In our study, despite being conducted in a population with a high component of individuals infected with HIV, we found that $16 \%$ (49 of 314) of all patients with tuberculosis were known to be HIV seropositive. Whilst the HIV status of all presumptively seronegative patients with tuberculous disease in this study was not determined, our data endorse the fact that most patients with tuberculosis are unlikely to have HIV infection. Examining the relationship from the opposite perspective, the proportion of AIDS patients who develop tuberculosis has been variously estimated at $4 \cdot 6 \%{ }^{9}$ and $6 \cdot 0 \%{ }^{10} \mathrm{We}$ have estimated that the prevalence of mycobacterial disease for all patients newly registered with HIV infection was $9 \cdot 6 \%$. If only local notification records had been assessed, the incidence amongst HIV patients would have been $0 \cdot 28 \%$ and this highlights an under reporting of tuberculosis infection in this group.

The role of $M$ avium complex infection in patients with HIV disease and, particularly, its impact on survival in these patients is controversial. ${ }^{11-14}$ In those individuals presumptively HIV negative, infection with $M$ avium alone occurred in $12.5 \%$ of patients ( 44 of 350 ) and in 91 of the $188 \mathrm{HIV}$ seropositive patients $(48 \%)$. These figures are surprisingly high. However, falsely negative HIV status, clinical relevance of certain cultures (for example, stool cultures), high incidence of alcoholism and malnutrition in the population studied, ethnicity, inclusion of $M$ scrofulaceum in the "MAIS" complex in the laboratory, and the diagnostic difficulties encountered with the biochemical identification methods used in the laboratory during the study period (compared with the currently used gene probe techniques) partly explain this finding. The HIV seropositive group was almost invariably culture positive during the later stages of AIDS and, given our finding of a four month mean survival following culture diagnosis of $M$ avium (data not shown), the likelihood is that many of these individuals were at the later stages of the disease process.

Equally, the significant incidence of extrapulmonary tuberculous infection in $25 \%$ of the HIV seronegative group is important. The explanation for this is not clear, and its relevance depends on the clinical setting. Falsely negative HIV seronegative status is a clinically important explanation. Notwithstanding this, these positive isolates might also be attributable to reactivation of old disease in ethnic groups and dissemination of infection in immunocompromised individuals who do not have HIV infection, both of which are relevant to this population.

In summary, this retrospective study highlights the trends in mycobacterial infection over a six year period at an inner London teaching hospital. The incidence of tuberculosis seems to be increasing in HIV seropositive and seronegative populations at a rate similar to that at other centres. Drug resistance does not appear to occur on the scale reported in the USA. The influence of HIV infection is complex. Prospective studies looking at known seropositive individuals and routine testing of presumptively seronegative patients with mycobacterial infection will allow further accurate evaluation of the impact of HIV.

1 Styblo K, Enarson DA. The impact of infection with human immunodeficiency virus on tuberculosis. In: Mitchell DM ed. Recent advances in respiratory medicine 5. Edinburgh: Churcill Livingstone, 1991:147-62.

2 Harries AD. Tuberculosis and human immunodeficiency virus infection in developing countries. Lancet 1990;335: 387-9. 
3 Barnes PF, Bloch AB, Davidson PT, Snider DE Jr. Tubermunodeficiency virus infection. N Engl f Med 1991:324:1644-50.

4 Spence DPS, Hotchkiss J, Williams CSD, Davies PDO. Tuberculosis and poverty. BMF 1993;307:759-61.

5 Raviglione MC, Sudre P, Rieder HL, Spinaci S, Kochi A. Secular trends of tuberculosis in Western Europe: A. Secular trends of tuberculosis in Western Europe: 1993;71:297-306.

6 Freiden TR, Sterling T, Pablos-Mendez A, Kilburn JO, Cauthen GM, Dooley SW. The emergence of drug resistant tuberculosis in New York City. N Engl f Med 1993; 328:521-6.

7 Collins $\mathrm{CH}$, Grange JM, Yates MD. Organization and practice in tuberculosis bacteriology. London: Butterworths, 1985.

8 Chaisson RE, Schecter EF, Theuer CP, Rutherford GW, Echenberg DF, Hopewell PC. Tuberculosis in patients with the acquired immunodeficiency syndrome. $A m R e v$ with the acquired immuno
Respir Dis 1987;136:570-4.

9 Watson JM, Meredith SK, Whitmore-Overton E, Bannister
B, Darbyshire JH. Tuberculosis and HIV: estimates of the overlap in England and Wales. Thorax 1993;48:199-203.

10 Helbert M, Robinson D, Buchanan D, Hellyer T, McCarthy $\mathbf{M}$, Brown I, et al. Mycobacterial infection in patients infected with the human immunodeficiency virus. Thorax 1990;45:45-8.

11 Horsburgh CR, Havlik JA, Ellis DA, et al. Survival of patients with acquired immunodeficiency syndrome and disseminated Mycobacterium avium complex infection with and without antimycobacterial chemotherapy. $A m$ Rev Respir Dis 1991;144:557-9.

12 Horsburgh CR Jr, Selik RM. The epidemiology of disseminated nontuberculous mycobacterial infection in acquired immunodeficiency syndrome (AIDS). $A m R e v$ Respir Dis 1989;139:4-7.

13 Chaisson RE, Hopewell PC. Mycobacteria and AIDS mortality. Am Rev Respir Dis 1989;139:1-3.

14 Horsburgh CR. Mycobacterium avium complex infection in the acquired immunodeficiency syndrome. $N$ Engl $f$ Med 1991;324:1332-8. 